\title{
Quasar Metal Abundances \& Host Galaxy Evolution
}

\author{
Fred Hamann ${ }^{1}$ and Leah E. Simon ${ }^{1}$ \\ ${ }^{1}$ Department of Astronomy, University of Florida, Gainesville, FL 32611-2055, USA \\ email: hamann@astro.ufl.edu
}

\begin{abstract}
High-redshift quasars provide a unique glimpse into the early evolution of massive galaxies. The physical processes that trigger major bursts of star formation in quasar host galaxies (mergers and interactions) probably also funnel gas into the central regions to grow the super-massive black holes (SMBHs) and ignite the luminous quasar phenomenon. The globally dense environments where this occurs were probably also among the first to collapse and manufacture stars in significant numbers after the big bang. Measurements of the elemental abundances near quasars place important constraints on the nature, timing and extent of this star formation. A variety of studies using independent emission and absorption line diagnostics have shown that quasar environments have gas-phase metallicities that are typically a few times solar at all observed redshifts. These results are consistent with galaxy evolution scenarios in which large amounts of star formation (e.g., in the central regions) precede the visibly bright quasar phase. An observed trend for higher metallicities in more luminmous quasars (powered by more massive SMBHs) is probably tied to the well-known mass-metallicity relation among ordinary galaxies. This correlation and the absence of a trend with redshift indicate that mass is a more important parameter in the evolution than the time elapsed since the big bang.
\end{abstract}

Keywords. galaxies: formation, galaxies: high-redshift, quasars: general, quasars: absorption lines, quasars: emission lines

\section{Introduction}

Quasars are powered by matter accreting onto super-massive black holes (SMBHs) with masses of order $10^{8}$ to $10^{10} \mathrm{M}_{\odot}$. During their brief lifetimes, just $10^{7}$ to $10^{8} \mathrm{yr}$, quasar bolometric luminosities easily exceed the total energy output from stars in the host galaxies. Most of this accretion-driven quasar activity occurred at high redshifts, in the range $z \sim 1-3$ but extending out to $z>6$ (Silverman et al. 2005, Richards et al. 2006). This is also the era when massive elliptical galaxies were rapidly being assembled and making most of their stars (Hopkins \& Beacom 2006). We see the results of this early evolution in the galaxies around us today. Giant ellipticals are gas poor with primarily old and metal-rich stellar populations. Their mean stellar metallicities are typically above solar, with the nuclear regions having $\langle Z\rangle \sim 2-3 \mathrm{Z}_{\odot}$ (Trager et al. 2000, Spolaor et al. 2009). The cores of giant spheroids also contain SMBHs that are mostly dark and dormant because their gaseous fuel supply has essentially run out. However, at early cosmic times, when the SMBHs were still growing and rapidly accreting matter, they were the engines of quasars. Every giant elliptical today was a quasar host at earlier times and higher redshifts.

The observed correlation between the masses of SMBHs and their surrounding spheroids (Tremaine et al. 2002) implies that SMBH growth in galactic nuclei is somehow connected to the same physical processes that make the galaxies. Remarkably, this physical connection occurs across spatial scales from $<1 \mathrm{pc}$ for the accreting SMBHs to $>1$ $\mathrm{kpc}$ in the galaxy hosts. One possibility is that "feedback" from the quasar, in the form of 
powerful outflows and perhaps ionizing radiation, directly regulates both star formation in the host galaxy and the growth of the SMBH itself (Kauffmann \& Haehnelt 2000).

A major goal of quasar research is to understand the evolutionary relationship between quasars, SMBHs and their host galaxies. The most basic question involves simply the relative timing of events: When does the quasar phase occur in relation to the major episodes of star formation and mass assembly in the host galaxy? One common view is that major mergers of gas-rich galaxies trigger both the growth of a central SMBH and bursts of star formation in the host that appear observationally as ultra-luminous infrared galaxies (ULIRGs, Sanders et al. 1988, Veilleux et al. 2009). Most of the SMBH growth occurs in obscurity, behind the dusty veil of the star-bursting ULIRG, but eventually a blowout of gas and dust reveals a visibly luminous quasar in the galactic center. In this scenario, the short-lived quasar phase is part of (and maybe an active participant in) the transition from the early stages of violent star formation to the more relaxed and passively evolving galaxy that comes later (also Hopkins et al. 2005).

Measurements of the elemental abundances near quasars provide important tests of the evolution. For example, the metallicities can tell us the extent to which star formation and chemical enrichment occurred in different environments prior to the observed quasar epoch. Specific abundance ratios, such as $\mathrm{Fe} / \alpha$, can further constrain the nature and timing of this star formation. The first review of quasar abundance studies appeared in Hamann \& Ferland (1999), which we updated most recently in Hamann et al. (2004 and 2007). Here we present a brief summary of the main results with further updates. Please see our previous reviews for more extensive discussions of earlier work.

\section{Metallicity Diagnostics and Results}

The various emission and absorption features in quasar spectra provide opportunities to measure the metal abundances across a wide of enviroments, from within $\sim 1 \mathrm{pc}$ of the central object to $>100 \mathrm{kpc}$ away in the outer host galaxies. Combining results from different diagnostics has the potential to map out the abundance distributions around quasars. It also provides the best hedge against the uncertainties in any particular technique. The sections below discuss spectral line diagnostics at rest-frame UV through visible wavelengths.

\subsection{Broad Emission Lines}

Broad emission lines (BELs) are present in essentially all quasars. They are also relatively easy to measure, which makes them ideal for studies of large quasar samples that can search for correlations between the abundances and other properties of the quasars (e.g., redshift, luminosity, SMBH mass, etc.) or their host galaxies (mass, morphology, star formation rate, cluster environment, etc.). The main challenge for BEL abundance work is that the metal lines cannot become uniformly stronger as the metallicity increases above a few percent of solar. This is because the metal lines control the cooling in this regime and such behavior would violate the balance between the energy absorbed and emitted by photoionized BEL clouds. Nonetheless, the effects of metallicity are imprinted on observed BEL spectra.

The most recent theoretical efforts to quantify these effects (Hamann et al. 2002, Nagao et al. 2005) consider that the BELs arise from distributions of clouds that span a range of densities and distances from the continuum source. This approach based on cloud distributions leads to general results that match observed quasar spectra quite well and avoid most of the uncertainties in other non-abundance cloud parameters. A further simplification in the calculations is that the metal abundances are scaled from 


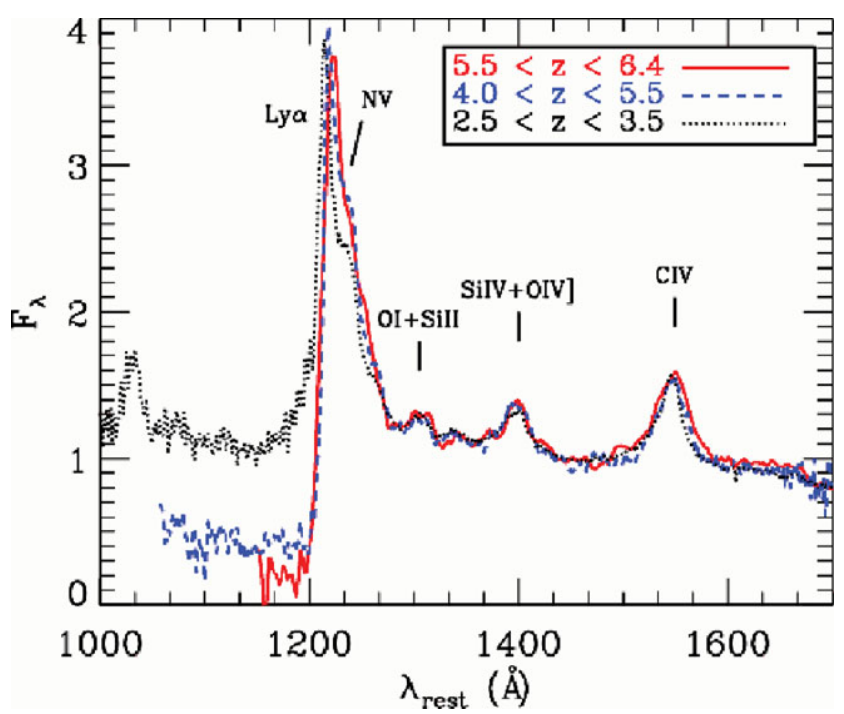

Figure 1. Mean spectra of quasars in three redshift intervals show the lack of evolution in the broad emission line ratios and thus the inferred metallicities (from Juarez et al. (2009).

the solar values $\dagger$, with nitrogen treated as a secondary element ( such that $\mathrm{N} / \mathrm{O} \propto \mathrm{O} / \mathrm{H}$ ). Hamann et al. described the resulting strong metallicity dependence of several UV line ratios involving nitrogen, such as $\mathrm{Nv} / \mathrm{C}$ IV, $\mathrm{Nv} /(\mathrm{C} \mathrm{IV}+\mathrm{O}$ VI) and $\mathrm{NIII}] / \mathrm{O}$ III]. Nagao et al. additionally promoted the use of other ratios, such as $(\mathrm{Si} \mathrm{IV}+\mathrm{O}$ IV] $) / \mathrm{C}$ IV, whose metallicity dependence appears to arise from a combination of saturation effects and weaker lines carrying a greater share of the total cooling as $Z$ increases.

The main result from these analyses is that quasar BEL regions have metallicities of typically 2-5 times solar at all redshifts (see also our reviews, Jiang et al. 2007, Juarez et al. 2009). It has been noted that different BEL ratios can indicate different metallicities by up to a factor of $\sim 2$ in the same quasar (Dietrich et al. 2003, Nagao et al. 2005). The best strategy is to take the average metallicity inferred from several line ratios. The worst case disparities between the line ratios might be a reasonable estimate of the theoretical uncertainties in individual quasars. However, there are no obvious biases that would push the derived metallicities higher, and therefore the general result for super-solar metallicities should be secure. Also, our ability to measure abundance trends in quasar samples should be much better than the uncertainties in individual estimates.

Another important result is that there is no significant trend in the BEL metallicities with redshift out to at least $z \sim 6$ (Figure 1; Nagao et al. 2005, Dietrich et al. 2003). There is, however, a significant trend with quasar luminosity that appears to have its origins in an even stronger positive correlation between the metallicity and SMBH mass (Warner et al. 2003 and in prep.). If we consider that more massive SMBHs reside in more massive galaxies, then the quasar mass-metallicity correlation is probably just another manifestation of the well-known mass-metallicity relation among galaxies.

In the first attempt to compare quasar metallicities directly to host galaxy properties, Simon \& Hamann (2009 and this proceedings) looked for trends with $L_{F I R}$ as a surrogate for the star formation rate in the hosts. They found none. Visibly bright quasars are generically metal-rich. The quasars associated with the most extreme star formation

$\dagger$ The metallicities reported in these papers should be scaled downward by $30 \%$ to reflect the most recent solar abundances (see Dhanda et al. 2007). The numbers here include this scaling. 
rates, with $L_{F I R}$ in the range of ULIRGs, do not appear to be signifantly younger or chemically less mature based on their BEL metallicities. This result is consistent with evolutionary schemes that have most of the star formation in the (inner) host galaxies occurring before the visible quasar epoch.

It should be noted that the BEL results apply strictly to the gas within $\sim 1 \mathrm{pc}$ of the central quasars. However, the star formation that produces the BEL metallicities probably occurs on much larger scales. If BEL regions are continuously replenished by gas from the accretion disk, then the total amount of metal-rich gas probed by the BELs is at least equal to the amount that funnels through the disk during a quasar's lifetime. This is roughly the black hole mass or $\geqslant 10^{9} \mathrm{M}_{\odot}$ for luminous quasars. This amount of gas with $Z \geqslant 2 \mathrm{Z}_{\odot}$ nominally requires enrichment by a stellar population with mass $\geqslant 10^{10} \mathrm{M}_{\odot}$ (assuming a normal Galactic IMF, see Hamann et al. 2007). Thus it appears that at least Galactic-bulge-size stellar populations are already present or in their final stages of assembly at the observed quasar epochs.

A more specific constraint on the ages of the stellar populations around quasars might be gained from measurements of the $\mathrm{Fe} / \alpha$ abundance. The age constraint comes from the $\sim 0.3$ to 0.5 Gyr delay in the Fe enrichment from SN Ia. Attempts to measure this ratio in quasars using their Fe II/Mg II BELs are poorly calibrated in an absolute sense and comparisons between quasars should probably be controlled for non-abundance parameters like luminosity and perhaps Eddington ratio $L / L_{\text {edd }}$ (Dong et al. 2009). Nonetheless, it has been argued that the lack of an observed trend in this line ratio with redshift means that SN Ia's are contributing at all epochs and therefore the bursts of star formation that enriched the BEL regions started at least 0.3 Gyr before the observed quasar redshifts (Dietrich \& Hamann 2008, Jiang et al. 2007 and refs. therein).

\subsection{Narrow Emission Lines}

Narrow emission lines probe much larger scales than the BELs, from roughly 100 to $10^{4} \mathrm{pc}$ around luminous quasars. The metal abundances in these regions have been measured in two ways. One approach is to use the usual $\mathrm{H}$ I and forbidden lines at visible wavelengths that are also used to classify starbursts and assorted AGN. At high redshifts, these lines present observational difficulties because they appear in the observed-frame infrared. In one large study involving 23000 low-redshift Seyfert 2 galaxies, which have much lower luminosities and than quasars, Groves et al. (2006) found the typical metallicities to be 2-4 $\mathrm{Z}_{\odot}$, consistent with earlier work on similar objects.

A second approach is to use the same UV line ratios as the BEL studies. This is possible only in sources where the BELs are weak or absent, e.g., in type 2 AGN. Nagao et al. (2006) used this approach to derive metallicities from the narrow UV lines in a sample of high-redshift type 2 quasars and radio galaxies. They found a larger range of results than Groves et al., but super-solar metallicities still appear to be typical.

All of the narrow emission line studies so far rely on calculations that choose a particular gas density, even though the actual densities are poorly known. It would be helpful to check these results against calculations that use a range of gas densities in cloud distributions, similar to the BEL studies discussed above.

\subsection{Broad Absorption Lines}

Broad absorption lines (BALs) form in massive outflows from the inner accretion disk. Figure 2 shows one example of a quasar BAL spectrum. Part of the motivation for studying BAL outflows is that they might play a role in the kinetic energy feedback that affects SMBH growth and the host galaxy evolution $(\S 1)$. Unfortunately, BAL studies are plagued by large uncertianties in the derived column densities due to line blending. 


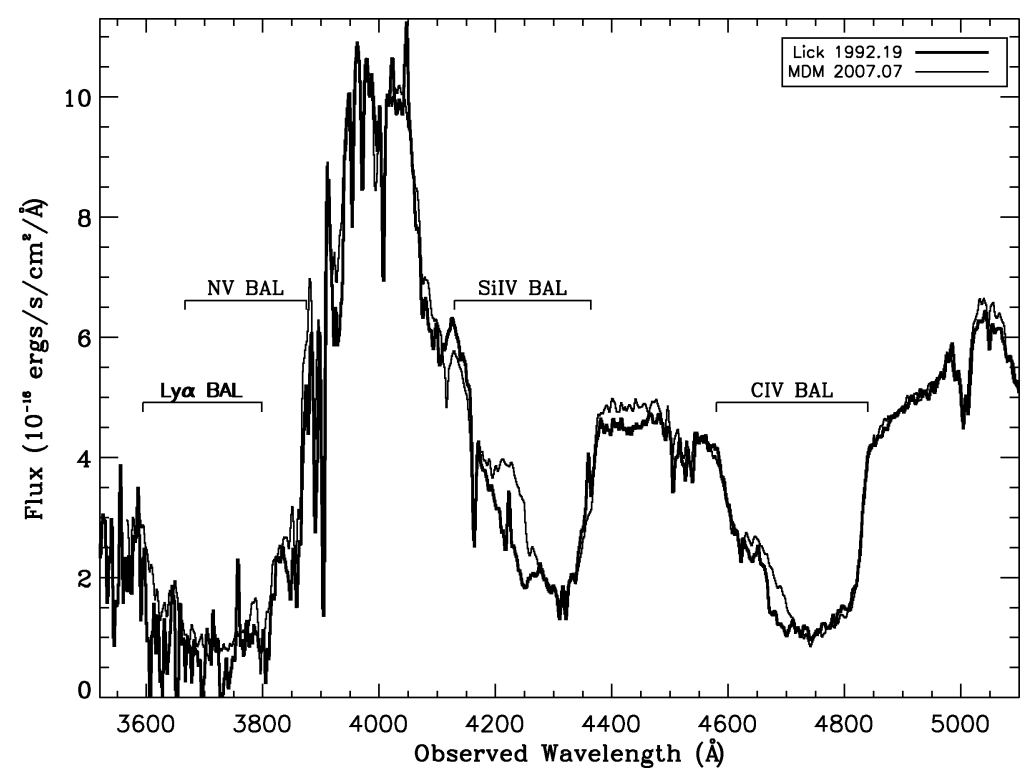

Figure 2. Rest-frame UV spectra of a redshift 2.4 quasar showing modest variability and some blending effects in its broad absorption lines (from Capellupo et al. in prep.).

In the best effort so far to measure the abundances in a BAL flow, Arav et al. (2001) used constraints on many lines to derive a metallicity consistent with solar. However, the uncertainties are still large and their finding for an enhanced phosphorus abundance suggests that the actual metallicity is several times larger than solar (Hamann 1998).

\subsection{Narrow Absorption Lines}

Narrow absorption lines (NALs, with profile widths less than a few hundred $\mathrm{km} \mathrm{s}^{-1}$ ) can form in a wide range of quasar environments, from outflows like the BALs to much more distant clouds in the outer host galaxies. They can also form in cosmologically gas that has no relation to the quasars whatsoever. Figure 3 shows a mixture of NALs in one quasar spectrum. The outflow lines (labeled A-E) were identified by their variable strengths, line profiles that are broad and smooth compared to the thermal speed and doublet ratios in $\mathrm{C}$ IV and $\mathrm{O}$ VI that reveal partial covering of the background continuum source $(<0.01$ pc across, Hamann et al. in prep.).

The theoretical steps in the abundance analysis are straightforward. They involve only an ionization correction applied to the measured ionic column densities. Even without an estimate of the ionization (e.g., if just one metal ion is measured) we can still derive useful lower limits on the metal/hydrogen abundance ratios (Hamann \& Ferland 1999). The main difficulties are usually more practical. Line saturation and possibly inhomogeneous column density distributions across the background emission source(s) can affect the reliability and interpretation of the derived column densities. However, these concerns can be addressed by analyzing (or fitting) several lines together and making reasonable assumptions about the spatial distribution of the absorbing gas (Hamann \& Sabra 2004, Arav et al. 2005, Gabel et al. 2005).

The particular outflow systems shown in Figure 3 indicate a metallicity of roughly $Z \sim 2 \mathrm{Z}_{\odot}$ with large uncertainties owing to poor constraints on the $\mathrm{H}$ I column. More reliable analyses of multiple outflow NALs in two other quasars (Arav et al. 2007, Gabel et al. 2006) yield $Z \sim 2-9 \mathrm{Z}_{\odot}$. Simon \& Hamann (2009 and in prep.) are conducting a 


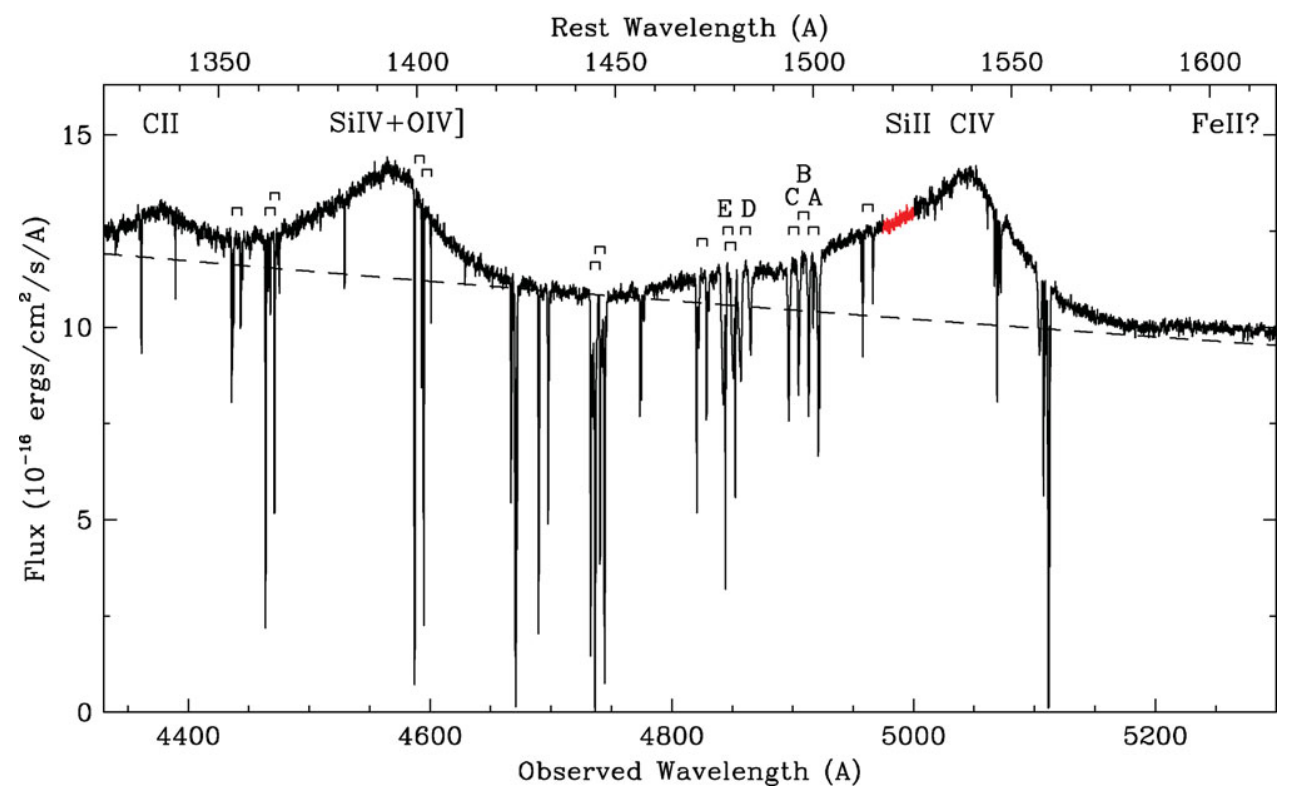

Figure 3. Rest-frame UV spectra showing a variety of narrow C IV absorption doublets marked by the downward facing brackets above the spectrum. The outflow systems, labelled A-E, have velocities from -8200 to $-12500 \mathrm{~km} \mathrm{~s}^{-1}$. Various broad emission lines are noted across the top (from Hamann et al. 2009).

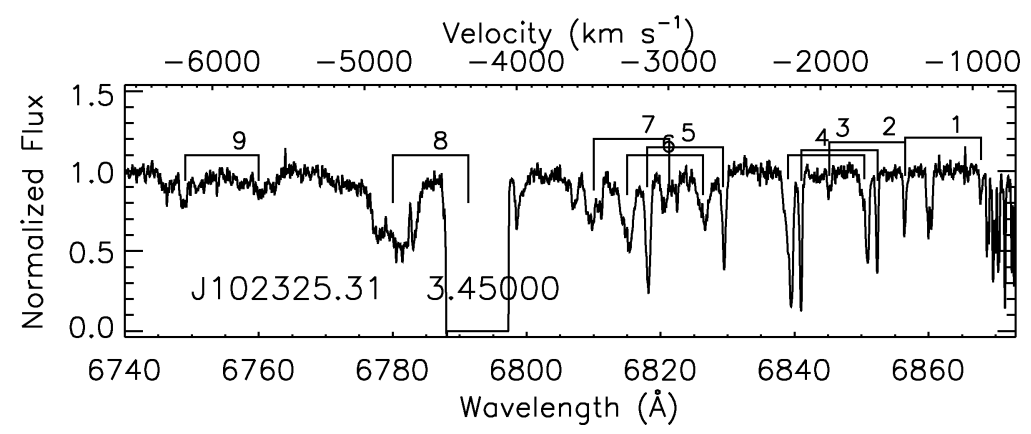

Figure 4. A complex of $9 \mathrm{C}$ IV NALs (labeled above the spectrum) at velocity shifts from roughly -1400 to $-6200 \mathrm{~km} \mathrm{~s}^{-1}$ in a quasar at redshift 3.4 (from Simon \& Hamann 2009).

survey of low-velocity NALs to derive metallicities and other properties of these absorbers in quasars at redshifts from $\sim 2$ to 4 .7. Figure 4 shows the diverse C IV systems measured in one particular quasar. This study emphasizes high redshifts to examine any redshift effects and obtain accurate H I column densities using ground-based measurements of several lines in the H I Lyman series. A significant fraction of the observed NAL systems (e.g., in Fig. 4) clearly form in a quasar outflow based on their broad profiles and evidence for partial covering. The best-measured systems in our survey so far have $Z \sim 2-4 \mathrm{Z}_{\odot}$, with a few outliers (that might be unrelated to the quasars) having sub-solar $Z$.

Overall, the NAL abundances agree with other quasar data. The metallicities tend to be a few times solar in the confirmed or suspected NAL outflow systems, and there is no evidence for a trend with redshift (see Hamann et al. 2007 and refs. therein). 


\section{Other Indicators of Star Formation in Quasar Hosts}

There are two key results in this broad research area that have a direct bearing on our quasar metallicity discussion. First, many normal, visibly luminous quasars have massive amounts of star formation ongoing in their host galaxies. For example, roughly $30 \%$ of high-redshift quasars have ULIRG-like FIR luminosities powered by star formation (Wang et al. 2008, Cox et al. 2005, Beelen 2006). The FIR luminosities and inferred star formation rates tend be lower around low-redshift quasars (Netzer et al. 2007), but this comparison might be affected by selection biases favoring more luminous sources at higher redshifts. Second, the dust masses that produce the strong FIR emissions are also large, of order $10^{8}$ to $10^{9} \mathrm{M}_{\odot}$ (see refs. above), requiring substantial amounts of star formation and metal enrichment before the observed quasar epochs. If the gas metallicity is solar with a normal gas/dust ratio, then the total mass of metal-rich gas accompanying this dust is $\sim 10^{10}$ to $10^{11} \mathrm{M}_{\odot}$. The stellar population needed to produce that amount of enriched gas is of order a few $\times 10^{10}$ to $10^{12} \mathrm{M}_{\odot}$ (assuming a Galactic IMF, Hamann et al. 2007).

Finally, it is worth mentioning that the gas-phase metallicities derived for ULIRGs are roughly in the range 0.5 to $3 \mathrm{Z}_{\odot}$, with the larger values believed to represent the most heavily obscured regions deep inside the galaxies (Veilleux et al. 2009, Rupke et al. 2008). These embedded regions are also where SMBHs might be growing during the ULIRG phase, to be revealed later as optically luminous (and metal-rich) quasars.

\section{Understanding Quasar Metal Abundances}

Quasar metallicities provide unique constraints on the relationship between SMBH growth and massive galaxy evolution. The general result for $Z \geqslant \mathrm{Z}_{\odot}$ near quasars is consistent with standard evolutionary models of elliptical galaxies, which today have old stellar populations with mean metallicities of $2-3 \mathrm{Z}_{\odot}$ in their cores $(\S 1)$. The metal-rich gas that created these stars might have been long ago expelled from the galaxies, consumed by the central SMBHs or used up in making stars, but we are probably measuring this gas directly in the environments of high-redshift quasars. The particular evolutionary stage probed by the quasar data must be after there is enough star formation to enrich that the gas to $Z \geqslant \mathrm{Z}_{\odot}$ but before the consumption or blowout of metal-rich gas is complete.

The amount of star formation that occurred before the quasar epoch is not directly constrained by the abundance data. However, simple arguments ( $\$ 2.1)$ indicate that the mass of metal-rich gas is at least as large as the $\mathrm{SMBH}$, or $\sim 10^{9} \mathrm{M}_{\odot}$ for luminous quasars, and therefore the mass of the enriching stellar population is $>10^{10} \mathrm{M}_{\odot}$. The percentage of gas converted into stars is more directly constrained by the metallicity. For example, in a simple "closed box" situation $>70 \%$ of the initial gas mass must be converted to stars to reach metallicities of several times solar (Hamann et al. 2007). More complicated enrichment schemes that include the transport of gas into and out of the galaxies must process a similarly high percentage of the gas into stars to reach high $Z$.

The quasar abundance results overall appear to support the evolutionary sequence outlined in $\S 1$, where the visible quasar phase appears generally after, or near the end of, a major ULIRG/starburst episode of star formation (§1). Some recent numerical simulations describe this sequence in detail (Hopkins et al. 2005, Di Matteo et al. 2004, Granato et al. 2004). By the time the central quasar is visibly revealed, after most of the star formation is complete, the gas-phase metallicities in the nuclear regions and in other dense pockets of star formation have reached 2-3 $\mathrm{Z}_{\odot}$ (Di Matteo et al. 2004, Li 
et al. 2007). The entire process, from the onset of the merger/starburst to the end of the quasar phase, can take $<0.5$ Gyr.

The absence of a trend in the observed quasar metallicities with redshift means that this rapid evolutionary sequence occurs in approximately the same way at all redshifts. The positive correlation between SMBH mass and metallicity near quasars (§2.1), akin to the well-known mass-metallicity relation among galaxies, suggests further that mass is a more important parameter in this evolutionary sequence than the time elapsed since the big bang.

We are grateful to Daniel Capellupo for help with Figure 2 and to NASA for financial support through the HST guest observer and Chandra theory programs.

\section{References}

Arav, N., et al. 2001, ApJ, 561, 118

Arav, N., Kaastra, J., Kriss, G. A., Korista, K. T., Gabel, J., \& Proga, D. 2005, ApJ, 620, 665

Arav, N., et al. 2007, ApJ, 658, 829

Beelen, A., et al. 2006, ApJ, 642, 694

Cox, P., et al. 2005, ESA-SP, 577, 115

Dhanda, N., Baldwin, J. A., Bentz, M. C., \& Osmer, P. S. 2007, ApJ, 658, 804

Di Matteo, T., Croft, R. A. C., Springel, V., \& Hernquist, L. 2004, ApJ, 610, 80

Dietrich, M., et al. 2003, ApJ, 589, 722

Dietrich, M. \& Hamann, F. 2008, RMxAC, 32, 65

Dong, X., Wang, J., Wang, T., Wang, H., Fan, X., Zhou, H., \& Yuan, W. 2009, arXiv:0903.5020

Gabel, J. R., Arav, N., \& Kim, T.-S. 2006, ApJ, 646, 742

Gabel, J. R., et al. 2005, ApJ, 623, 85

Granato, G. L., De Zotti, G., Silva, L., Bressan, A., \& Danese, L. 2004, ApJ, 600, 580

Groves, B. A., Heckman, T. M., \& Kauffmann, G. 2006, MNRAS, 371, 1559

Hamann, F., Warner, C., Dietrich, M., \& Ferland, G. 2007, ASP-CS, 373, 653

Hamann, F., Dietrich, M., Sabra, B. M., \& Warner, C. 2004, COAS, 440

Hamann, F., Korista, K. T., Ferland, G. J., Warner, C., \& Baldwin, J. 2002, ApJ, 564, 592

Hamann, F. \& Ferland, G. 1999, ARAA, 37, 487

Hamann, F. 1998, ApJ, 500, 798

Hamann, F. \& Sabra, B. 2004, ASP-CS, 311, 203

Hopkins, A. M. \& Beacom, J. F. 2006, ApJ, 651, 142

Hopkins, P. F., et al. 2005, ApJ, 630, 705

Jiang, L., et al. 2007, $A J, 134,1150$

Juarez, Y., et al. 2009, AA, 494, L25

Kauffmann, G. \& Haehnelt, M. 2000, MNRAS, 311, 576

Li, Y., et al. 2007, ApJ, 665, 187

Nagao, T., Maiolino, R., \& Marconi, A. 2006, AA, 447, 863

Nagao, T., Marconi, A., \& Maiolino, R. 2006, AA, 447, 157

Netzer, H., et al. 2007, ApJ, 666, 806

Richards, G. T., et al. 2006, $A J, 131,2766$

Rupke, D. S. N., Veilleux, S., \& Baker, A. J. 2008, ApJ, 674, 172

Sanders, D. B., Soifer, B. T., Elias, J. H., Neugebauer, G., \& Matthews, K. 1988, ApJ, 328, L35

Silverman, J. D., et al. 2005, ApJ, 624, 630

Simon, L. E., \& Hamann, F. 2009, submitted to MNRAS

Spolaor, M., Proctor, R. N., Forbes, D. A., \& Couch, W. J. 2009, ApJ, 691, L138

Trager, S. C., Faber, S. M., Worthey, G., \& González, J. J. 2000, AJ, 120, 165

Tremaine, S., et al. 2002, ApJ, 574, 740

Veilleux, S., et al. 2009, ApJS, 182, 628

Wang, R., et al. 2008, ApJ, 687, 848

Warner, C., Hamann, F., \& Dietrich, M. 2003, ApJ, 596, 72 